MATHEMATICS OF COMPUTATION

Volume 66, Number 217, January 1997, Pages 237-260

S 0025-5718(97)00796-5

\title{
A GENERAL FRAMEWORK FOR HIGH-ACCURACY PARAMETRIC INTERPOLATION
}

\author{
KNUT MØRKEN AND KARL SCHERER
}

\begin{abstract}
In this paper we establish a general framework for so-called parametric, polynomial, interpolation methods for parametric curves. In contrast to traditional methods, which typically approximate the components of the curve separately, parametric methods utilize geometric information (which depends on all the components) about the curve to generate the interpolant. The general framework suggests a multitude of interpolation methods in all space dimensions, and some of these have been studied by other authors as independent methods of approximation. Since the approximation methods are nonlinear, questions of solvability and stability have to be considered. As a special case of a general result, we prove that four points on a planar curve can be interpolated by a quadratic with fourth-order accuracy, if the points are sufficiently close to a point with nonvanishing curvature. We also find that six points on a planar curve can be interpolated by a cubic, with sixth-order accuracy, provided the points are sufficiently close to a point where the curvature does not have a double zero. In space it turns out that five points sufficiently close to a point with nonvanishing torsion can be interpolated by a cubic, with fifth-order accuracy.
\end{abstract}

\section{INTRODUCTION}

The traditional approach to approximation of parametric curves is to consider the curve as a vector function and then apply a suitable scheme for approximation of functions to each component, see for example [7]. Recently, several authors (e.g., $[2,3,4,5,6,8,11,13,14,15,18,19,20,21,22])$ have developed schemes which in the approximation of one component uses information about the other components. Such methods of approximation are conveniently called parametric approximation methods. The main motivation for studying such methods is that they often give better accuracy for the same class of approximating functions. For example, it is well known that in approximation of functions, cubic polynomials are fourth-order accurate, while there are cubic parametric schemes that are sixth-order accurate for plane curves, see e.g. [2] (note that the interpolation scheme studied in [2] was also used in [11] for the approximation of offset-curves).

In this paper we develop a general framework for high-accuracy, parametric, polynomial interpolation methods. This unifies a number of seemingly disparate parametric interpolation schemes that have appeared in the literature, see $[2,11$, 13, 18, 19]. Traditional interpolation methods are applied to parametric curves

Received by the editor December 15, 1994 and, in revised form, November 21, 1995 and January 26, 1996.

1991 Mathematics Subject Classification. Primary 41A05, 41A10, 41A25, 65D05, 65D17; Secondary 65D10.

(C) 1997 American Mathematical Society 
simply by matching position and/or derivatives at the same parameter values, in general

$$
\boldsymbol{p}^{(j)}(t)=\boldsymbol{f}^{(j)}(t),
$$

where $\boldsymbol{f}$ (we use boldface type for vectors) is a given curve to be approximated by $\boldsymbol{p}$ by interpolation at the point $t$, and the superscript denotes the $j$ th derivative. Parametric interpolation methods are (implicitly or explicitly) based on the idea that we can allow $\boldsymbol{p}$ to approximate a reparametrized version of $\boldsymbol{f}$, i.e., we replace the interpolation condition (1) by

$$
\boldsymbol{p}^{(j)}(s)=D^{j}(\boldsymbol{f} \circ \phi)(s),
$$

where the operator $D$ denotes ordinary differentiation and $\phi$ is a change of parameter, i.e., a strictly increasing real function. Given a change of parameter $\phi$, we can determine a polynomial $\boldsymbol{p}$ by interpolation of the type above at a suitable number of points. We can then try to adjust $\phi$, and hence $\boldsymbol{p}$, such that $\boldsymbol{p}$ satisfies some desirable condition.

As an example, suppose we search for $\boldsymbol{p}$ among the quintic polynomials. We can then try to adjust $\phi$ so that $\boldsymbol{p}$ is reduced to a cubic polynomial. The scheme of de Boor, Höllig and Sabin (BHS) can be interpreted in this way; by a suitable choice of the change of parameter $\phi$, a quintic interpolating polynomial is reduced to a cubic one, without sacrificing the sixth-order accuracy. For space curves it is in general not possible to reduce quintics to cubics; however, one can in general reduce quartics to cubics and hence obtain cubic schemes that are fifth-order accurate.

In $\S 2$, we discuss different ways of measuring the error, and define the concept of approximation order for parametric approximation schemes, while in $\S 3$, we consider in detail interpolation conditions of the form (2). In $\S 4$ we show how the ideas of classical interpolation of functions can be generalized to parametric interpolation, while in $\S 5$ we prove solvability and stability of the simplest schemes of this type in each space dimension. $\S 6$ is devoted to a detailed study of cubic schemes in the plane, and we show that these schemes are always solvable and sixth-order accurate in the neighborhood of a point $\boldsymbol{f}(0)$ on a curve $\boldsymbol{f}$ that satisfies $\operatorname{span}\left\{\boldsymbol{f}^{\prime}(0), \boldsymbol{f}^{\prime \prime}(0), \boldsymbol{f}^{\prime \prime \prime}(0)\right\}=\mathbb{R}^{2}$, i.e., the matrix built from the first three derivatives of $\boldsymbol{f}$ should have rank 2. As a special case we conclude that the BHS-scheme is solvable and sixth-order accurate under weaker conditions than those given in $[2]$.

An inherent problem with nonlinear systems of equations is that there are in general many solutions, and the systems that arise in parametric interpolation are not exempted from this rule. This and related problems are not considered in this paper but must be addressed if the schemes that we suggest are to become practical alternatives for approximation of parametric curves. In [16] some of these questions are discussed in the special case of quadratic approximation of planar curves.

\section{Approximation order AND ERROR MEASURES}

Given a parametric curve $\boldsymbol{f}$ and an approximation $\boldsymbol{p}$, both defined on the same interval $I$, the traditional way to measure the error in the approximation is to take some norm of the difference $\boldsymbol{f}(t)-\boldsymbol{p}(t)$ (note that there is no loss of generality in 
assuming the two curves to be defined on the same interval). In the plane one can for instance use

$$
\begin{aligned}
\|\boldsymbol{f}-\boldsymbol{p}\| & =\max _{t \in I}|\boldsymbol{f}(t)-\boldsymbol{p}(t)| \\
& =\max _{t \in I}\left(\left(f_{1}(t)-p_{1}(t)\right)^{2}+\left(f_{2}(t)-p_{2}(t)\right)^{2}\right)^{1 / 2} .
\end{aligned}
$$

Here, the functions $f_{i}$ and $p_{i}$ for $i=1,2$ denote the components of $\boldsymbol{f}$ and $\boldsymbol{p}$. More generally, one can combine the usual $\ell^{q}$ and $L^{r}$-norms,

$$
\begin{aligned}
&\|\boldsymbol{f}-\boldsymbol{p}\|_{\ell^{q}, L^{r}}=\|\| \boldsymbol{f}-\boldsymbol{p}\left\|_{\ell^{q}}\right\|_{L^{r}} \\
&=\left(\int_{I}\left(\left|f_{1}(t)-p_{1}(t)\right|^{q}+\left|f_{2}(t)-p_{2}(t)\right|^{q}\right)^{r / q} d t\right)^{1 / r}, \\
&\|\boldsymbol{f}-\boldsymbol{p}\|_{L^{r}, \ell^{q}}=\left\|\left(\left\|f_{1}-p_{1}\right\|_{L^{r}},\left\|f_{2}-p_{2}\right\|_{L^{r}}\right)\right\|_{\ell^{q}},
\end{aligned}
$$

see [17], where general approximation theory for such norms is developed. The norm defined in (3) above clearly corresponds to the special case $q=2$ and $r=\infty$ in (4).

The advantage of determining an approximation $\boldsymbol{p}$ that is close to $\boldsymbol{f}$ in the sense that the norm in (3) is small for all $t$, is that the point $\boldsymbol{p}(t)$ will be close to $\boldsymbol{f}(t)$. If we are approximating a path for a camera for example, where $t$ measures time elapsed along the path, this is important. Often, however, it is only the geometric object represented by $\boldsymbol{f}$ that is important and not the particular parametrization. All we want is an approximation $\boldsymbol{p}$ such that the graphs, or point-sets, of $\boldsymbol{p}$ and $f$ are "close". A well-known metric for measuring such closeness is the Hausdorff metric, but unfortunately this metric does not lend itself very well to computations.

To obtain a geometric error measure that is more suitable for practical implementation on a computer, we use the idea of reparametrization. For purely geometric purposes it is more natural to compare $\boldsymbol{f}(t)$ with the point on the curve given by $\boldsymbol{p}$ that is as close as possible, or at least close to the closest point. This will be a point $\boldsymbol{p}(s)$ with parameter value $s$. As $t$ varies, we get different values of $s$, or $s=\phi(t)$ for some function $\phi$. Instead of (3), we therefore use an error measure on the form

$$
\max _{t \in I}|\boldsymbol{f}(t)-\boldsymbol{p}(\phi(t))| .
$$

This motivates the following definition of a metric on the set of parametric curves, see [14] for more details.

Definition 1. Let $\boldsymbol{f}$ and $\boldsymbol{g}$ be two parametric curves in $\mathbb{R}^{d}$, both defined on the same parameter interval $I=[a, b]$. Denote by $\mathbb{A}$ the set of infinitely differentiable increasing functions mapping $I$ onto itself, i.e.,

$$
\mathbb{A}=\left\{\phi \in C^{\infty}[a, b] \mid \phi(a)=a, \phi(b)=b \text { and } \phi^{\prime}>0\right\} .
$$

The distance between $\boldsymbol{f}$ and $\boldsymbol{g}$ is then defined by the metric

$$
d(\boldsymbol{f}, \boldsymbol{g})=d(\boldsymbol{f}, \boldsymbol{g})_{[a, b]}=\inf _{\phi \in \mathbb{A}} d_{\phi}(\boldsymbol{f}, \boldsymbol{g})=\inf _{\phi \in \mathbb{A}} \sup _{t \in I}|\boldsymbol{f}(t)-\boldsymbol{g}(\phi(t))|,
$$

where $|\cdot|$ denotes Euclidean distance in $\mathbb{R}^{d}$. 
The functions in $\mathbb{A}$ will be called allowable changes of parameter in what follows, and a curve $\boldsymbol{f}$ composed with some $\phi$ in $\mathbb{A}$ will be called a reparametrization $\boldsymbol{f} \circ \phi$ of $\boldsymbol{f}$. Which interval the functions in $\mathbb{A}$ are defined on will be clear from the context.

Note also that we could just as well have defined the metric by

$$
d(\boldsymbol{f}, \boldsymbol{g})=\inf _{\psi \in \mathbb{A}} \sup _{s \in I}|\boldsymbol{f}(\psi(s))-\boldsymbol{g}(s)|,
$$

since all the functions in $\mathbb{A}$ have inverses in $\mathbb{A}$ (the set $\mathbb{A}$ is a group under composition). That $d(\boldsymbol{f}, \boldsymbol{g})$ provides a metric on the set of (equivalence classes under reparametrization of) parametric curves is straightforward, see [14].

Degen $([3,4])$ has used error measures that are very similar to the metric $d$. In fact, his normal distance [3] is nothing but $d_{\phi}(\boldsymbol{f}, \boldsymbol{g})$, where the change of parameter $\phi$ is the function that assigns to a parameter value $t$ the parameter value $\phi(t)$ such that the normal at $\boldsymbol{f}(t)$ intersects $\boldsymbol{g}$ at $\boldsymbol{g}(\phi(t))$. In [4], he uses a similar change of parameter which is based on intersection with $\boldsymbol{f}^{\prime \prime}(t)$ instead of the normal at $\mathbf{f}(t)$. Note that in many cases, the Hausdorff distance between $\boldsymbol{f}$ and $\boldsymbol{g}$ will coincide with Degen's normal distance and therefore with $d(\boldsymbol{f}, \boldsymbol{g})$, see [3].

To study the positive effect that the metric $d$ has on error estimates, let $\boldsymbol{V}$ be a class of vector-valued functions by which we approximate a given curve $\boldsymbol{f}$ defined on the interval $I=[a, b]$. Then we obviously have

$$
\inf _{\boldsymbol{g} \in \boldsymbol{V}} d(\boldsymbol{f}, \boldsymbol{g})=\inf _{\boldsymbol{g} \in \mathcal{V}} d(\boldsymbol{f}, \boldsymbol{g}),
$$

where $\mathcal{V}$ denotes the larger class

$$
\mathcal{V}=\{\boldsymbol{g} \circ \phi \mid \boldsymbol{g} \in \boldsymbol{V} \text { and } \phi \in \mathbb{A}\} .
$$

The case where $\boldsymbol{V}$ is a space of polynomial functions (i.e., not vector-valued) and the change of parameter is restricted to be a polynomial has been studied by the Bulgarian School of Approximation, see [23, 25].

In our context, the class $\boldsymbol{V}$ can be thought of as a linear $C^{k}$-space of polynomial spline curves in $\mathbb{R}^{d}$, and $\mathcal{V}$ as a space of $G C^{k}$-splines or $\beta$-splines (see [1]), where the $\beta$ 's as well as the coefficients are free. We will however only consider methods for constructing each polynomial piece explicitly, i.e., local interpolation methods.

From (6) and (7) we see that the approximation power of $\boldsymbol{V}$ in the metric $d$ is the same as that of $\mathcal{V}$. We could therefore search for optimal approximation schemes $B$ by associating with each curve $\boldsymbol{f}$ a curve $\boldsymbol{g}=B(\boldsymbol{f})$ in $\boldsymbol{V}$ such that

$$
d(\boldsymbol{f}, B(\boldsymbol{f})) \leq C \inf _{\boldsymbol{g} \in \mathcal{V}} d(\boldsymbol{f}, \boldsymbol{g}),
$$

for some constant $C$ independent of $\boldsymbol{f}$. However, this appears rather complicated. In this paper our aim will instead be to construct schemes with high convergence order with respect to the metric $d$ in Definition 1 . To do this, we must first make clear what we mean by convergence order.

Definition 2. Let $H$ be some positive real number, let $f$ be a parametric curve defined in a neighborhood $I_{H}=\left[t_{0}-H, t_{0}+H\right]$ of $t_{0}$, and let $S$ be an approximation scheme that assigns to each $h<H$ an approximation $S_{h}(\boldsymbol{f})$ to the part of $\boldsymbol{f}$ defined on the interval $I_{h}$. Then the scheme $S$ is said to have (local) convergence order $m$ at $t_{0}$ if the inequality

$$
d\left(\boldsymbol{f}, S_{h}(\boldsymbol{f})\right)_{I_{h}} \leq C h^{m}
$$

holds for some constant $C$ independent of $h$. 
Throughout this paper we will usually assume that $t_{0}=0$, which causes no loss of generality.

In function approximation it is usually simple to check that the $C$ in an inequality of the type

$$
\left\|f-B_{h}(f)\right\| \leq C h^{m}
$$

is independent of $h$. The typical way of studying approximation order in terms of the metric $d$ is to establish an inequality of the type

$$
d_{\phi_{h}}\left(\boldsymbol{f}, S_{h}(\boldsymbol{f})\right) \leq \max _{\theta \in(-h, h)}\left|D^{m}\left(\boldsymbol{f} \circ \phi_{h}\right)(\theta)\right| h^{m},
$$

for some suitable $\phi_{h} \in \mathbb{A}$. To get from (9) to an inequality of the form (8), it is therefore important to bound $\phi_{h}$ and its first $m$ derivatives independently of $h$. This motivates the following definition of stability.

Definition 3. A family of parameter changes $\left\{\phi_{s}\right\}_{s}$ that depend on the parameters $\boldsymbol{s}=\left(s_{i}\right)_{i=1}^{m}$ is said to be stable of order $r$ at $z$ if the first $r$ derivatives of $\phi_{\boldsymbol{s}}$ remain uniformly bounded as the $s_{i}$ coalesce at $z$. In other words, for stability there must exist some real number $C$ such that

$$
\lim _{\substack{s_{i} \rightarrow z \\ i=1, \ldots, m}} \max _{s \in\left[s_{1}, s_{m}\right]}\left|\phi_{\boldsymbol{s}}^{(j)}(s)\right| \leq C \quad \text { for } j=0,1, \ldots, r .
$$

We will discuss the problem of stability in more detail in $\S 5$.

How can we find schemes that satisfy inequalities of the type (9)? One possibility is to follow the same recipe as in function approximation, and let $S_{h}$ be accurate for polynomials of degree $m-1$. Since (9) by definition is equivalent to

$$
\max _{t \in[-h, h]}\left|\left(\boldsymbol{f} \circ \phi_{h}\right)(t)-S_{h}(\boldsymbol{f})(t)\right| \leq \max _{\theta \in(-h, h)}\left|D^{m}\left(\boldsymbol{f} \circ \phi_{h}\right)(\theta)\right| h^{m},
$$

this means that $S_{h}$ must reproduce the degree- $m-1$ Taylor expansion of some reparametrization of $\boldsymbol{f}$, and that $\left\{\phi_{h}\right\}$ must be stable of order $m$ as $h$ tends to zero. But except for stability, the change of parameter $\phi_{h}$ is arbitrary, and this illustrates once again the extra flexibility that is gained by employing the metric $d$.

How can we make use of this extra flexibility? Let $\phi_{h}$ be some as yet unspecified change of parameter for $\boldsymbol{f}$ on the interval $[-h, h]$. We then choose $S_{h}(\boldsymbol{f})=$ $T_{h}\left(f \circ \phi_{h}\right)$, where $T_{h}$ is a traditional, linear approximation scheme that reproduces polynomials of degree $m-1$. One possibility is for example to let $T_{h}$ denote interpolation at $m$ points in $[-h, h]$. With $T_{h}$ fixed, we can try to determine a specific $\phi_{h}$ that gives $S_{h}(\boldsymbol{f})$ some desirable property. In this paper, our aim will be to determine some $\phi_{h}$ that reduces the degree of the polynomial $S_{h}(\boldsymbol{f})$. For example, if all the $m$ interpolation points are chosen at $t=0$ (Taylor expansion), we will search for $\phi_{h}$ such that $D^{m-i}\left(\boldsymbol{f} \circ \phi_{h}\right)(0)=0$ for $i=1, \ldots, \ell$, with $\ell$ as large as possible. Note that these equations do not involve the approximating polynomial at all.

Rewriting the left-hand side of (10) in the equivalent form

$$
\max _{s \in[-h, h]}\left|\boldsymbol{f}(s)-\left(S_{h}(\boldsymbol{f}) \circ \phi_{h}^{-1}\right)(s)\right|,
$$

with $\phi_{h}^{-1}$ the inverse of $\phi_{h}$, makes clear the interpretation of the construction of $S_{h}$ as approximation from the set $\mathcal{V}$, see also (6). In this form, the determination of 
$\phi_{h}$ and $S_{h}(\boldsymbol{f})$ becomes more intertwined. This is the approach taken by Degen [4] and others.

Another variation of the same principle is to construct parametrization-invariant schemes by interpolating parametrization-invariant quantities like position, tangent direction and curvature at a number of points along the curve. Suppose the curve is given in some parametrization as $\boldsymbol{f}$; then the abovementioned quantities are nothing but position, first and second derivatives of the curve in arc length parametrization. But since the metric we use to measure the error does not differentiate between different parametrizations of $\boldsymbol{f}$, we see that schemes constructed in this way add nothing new to what we have said already. The scheme in [2] and [11] is indeed equivalent to a quintic scheme which by reparametrization is reduced to a cubic scheme.

It should be noted that the advantage of using the metric $d$, i.e., allowing reparametrization, is not restricted to polynomials, the class $\boldsymbol{V}$ in (6) is arbitrary. It does of course remain to be seen how much the quality of the approximation can be improved in passing from $\boldsymbol{V}$ to $\mathcal{V}$. Here we will try to answer this question for some polynomial schemes. In $[4,22]$, rational schemes are discussed.

\section{REPARAMETRIZED INTERPOLATION CONDITIONS}

Our main interest in this paper is to construct parametric interpolation schemes with high approximation order. As we have already mentioned, the idea is to replace a traditional interpolation condition like (1) by the more general condition

$$
\boldsymbol{p}^{(j)}(s)=D^{j}(\boldsymbol{f} \circ \phi)(s),
$$

and then choose $\phi$ in some clever way. Here $f$ is a given curve, the change of parameter is given by $\phi$, and $\boldsymbol{p}$ is the unknown interpolating polynomial. The following well-known lemma reveals some of the structure of conditions like (11).

Lemma 4. Let $\boldsymbol{f}$ be a given parametric curve, let $\left(y_{i}\right)$ be $M$ distinct interpolation points, suppose that $\ell_{i}$ derivatives are to be interpolated at $y_{i}$, let $\phi$ be an allowable change of parameter, set $\boldsymbol{g}=\boldsymbol{f} \circ \phi$ and let $x_{i}$ be given by $y_{i}=\phi\left(x_{i}\right)$. Let $\boldsymbol{p}$ denote a polynomial of degree $m-1$, where $m=\sum_{i=1}^{M} \ell_{i}$, that satisfies the interpolation conditions

$$
\boldsymbol{p}^{(j)}\left(x_{i}\right)=\boldsymbol{g}^{(j)}\left(x_{i}\right) \quad \text { for } j=0,1, \ldots, \ell_{i}-1 \text { and } i=1, \ldots, M .
$$

The interpolation conditions at $x_{i}$ can then be written as $\boldsymbol{p}_{i}=A_{i} \boldsymbol{f}_{i}$ where

$$
\begin{aligned}
\boldsymbol{p}_{i} & =\left(\boldsymbol{p}\left(x_{i}\right), \boldsymbol{p}^{\prime}\left(x_{i}\right), \ldots, \boldsymbol{p}^{\left(\ell_{i}-1\right)}\left(x_{i}\right)\right) \quad \text { and } \\
\boldsymbol{f}_{i} & =\left(\boldsymbol{f}\left(y_{i}\right), \boldsymbol{f}^{\prime}\left(y_{i}\right), \ldots, \boldsymbol{f}^{\left(\ell_{i}-1\right)}\left(y_{i}\right)\right),
\end{aligned}
$$

and $A_{i}$ is a lower triangular matrix on the form

$$
A_{i}=\left(\begin{array}{ccccc}
1 & 0 & 0 & \ldots & 0 \\
0 & \beta_{i, 1} & 0 & \ldots & 0 \\
0 & \beta_{i, 2} & \beta_{i, 1}^{2} & \ldots & 0 \\
\vdots & \vdots & \vdots & \ddots & \vdots \\
0 & \beta_{i, \ell_{i}-1} & \ldots & \ldots & \beta_{i, 1}^{\ell_{i}-1}
\end{array}\right)
$$

with $\beta_{i, j}=\phi^{(j)}\left(x_{i}\right)$. 
The complete interpolation conditions (12) can be enforced by requiring $\boldsymbol{F}=A \boldsymbol{P}$, where

$$
\boldsymbol{P}=\left(\boldsymbol{p}_{1}, \ldots, \boldsymbol{p}_{M}\right) \quad \text { and } \quad \boldsymbol{F}=\left(\boldsymbol{f}_{1}, \ldots, \boldsymbol{f}_{M}\right)
$$

and $A$ is the block diagonal matrix $A=\operatorname{diag}\left(A_{1}, \ldots, A_{M}\right)$.

A matrix on the form (13) is called a connection matrix, see [10]. The entries of a connection matrix are given by Faa di Bruno's formula, see [12, p. 50]. We record this in a lemma, and use the formula in a form that can be found in [9].

Lemma 5. Let $\boldsymbol{f}$ be a parametric curve, let $\phi$ be a change of parameter, and set $\alpha_{k}=D^{k}(\phi(0))$. Then

$$
D^{k}(\boldsymbol{f} \circ \phi)(0)=\sum_{j=1}^{k} a_{k, j} \boldsymbol{f}^{(j)}(0)
$$

where

$$
a_{k, j}=\sum_{\substack{k_{1}+k_{2}+\cdots+k_{j}=k \\
k_{l}>0, l=1, \ldots, j}}\left[\begin{array}{c}
k \\
k_{1}, k_{2}, \ldots, k_{j}
\end{array}\right] \alpha_{k_{1}} \alpha_{k_{2}} \cdots \alpha_{k_{j}}
$$

and

$$
\left[\begin{array}{c}
k \\
k_{1}, k_{2}, \ldots, k_{j}
\end{array}\right]=\frac{k !}{k_{1} ! k_{2} ! \cdots k_{j} ! m_{1} ! m_{2} ! \cdots m_{r} !} .
$$

For each set of integers $k_{1}, \ldots, k_{j}$, the integer $r$ denotes the number of distinct integers in the set, and $m_{1}, \ldots, m_{r}$ denotes the number of times each of these distinct integers occurs among $k_{1}, \ldots, k_{j}$.

Lemma 4 reduces the problem of working with $\phi$ to that of handling the $\beta$ parameters. Note, however, that the $\beta$ 's do not determine a change of parameter $\phi$ completely; they just prescribe $\phi$ and its first derivatives at the points $\left(x_{i}\right)_{i=1}^{M}$. The next result (which we expect is known) guarantees that if the $\beta$ 's are reasonable, then there exists a valid change of parameter $\phi$ which interpolates the $\beta$ 's with value $y_{i}$ at the $x_{i}$.

Lemma 6. Suppose that the $y_{i}$, the $x_{i}$ and the $\beta$-parameters are given and satisfy $x_{i}<x_{i+1}$ and $y_{i}<y_{i+1}$ for $i=1,2, \ldots, M-1$, and $\beta_{i, 1}>0$ for $i=1,2, \ldots$, $M$. Then there is a $C^{\infty}$ allowable change of parameter $\phi$ with $\phi^{\prime}(t)>0$ for all $t \in\left[x_{1}, x_{M}\right]$ that satisfies the interpolation conditions

$$
\begin{aligned}
\beta_{i, 0} & =y_{i}=\phi\left(x_{i}\right), \\
\beta_{i, 1} & =\phi^{\prime}\left(x_{i}\right), \\
\beta_{i, 2} & =\phi^{\prime \prime}\left(x_{i}\right), \\
\vdots & \\
\beta_{i, \ell_{i}} & =\phi^{\left(\ell_{i}\right)}\left(x_{i}\right)
\end{aligned}
$$

for $i=1,2, \ldots, M$.

Proof. The lemma simply says that if the data are increasing, then there is also an increasing $C^{\infty}$-solution to the interpolation problem.

Let $\hat{\phi}$ be a polynomial that satisfies the interpolation conditions (15). If $\hat{\phi}^{\prime}(t)>0$ for all $t$ in $\left[x_{1}, x_{M}\right]$ we can clearly set $\phi=\hat{\phi}$. Otherwise, there is at least one interval 
$I_{i}=\left(x_{i}, x_{i+1}\right)$ with $\hat{\phi}^{\prime}(t)<0$ for some $t$ in $I_{i}$. Note however that in a neighborhood of both $x_{i}$ and $x_{i+1}$ we have $\hat{\phi}^{\prime}>0$. The idea of the proof is to add to $\hat{\phi}$ a function $\psi$ with support in $(a, b)$, where $x_{i}<a<b<x_{i+1}$, such that the sum $\phi=\hat{\phi}+\psi$ satisfies the interpolation conditions (15) and is strictly increasing in $I_{i}$.

The monotonicity requirement of $\hat{\phi}+\psi$ is equivalent to $\psi^{\prime}(t)>-\hat{\phi}^{\prime}(t)$ for $t \in I_{i}$. Now let $a$ and $b$ be the smallest and largest zeros of $\hat{\phi}^{\prime}$ in $I_{i}$ respectively. Since the $C^{\infty}$-functions are dense in $C[a, b]$ (equipped with the sup-norm) and since $\hat{\phi}^{\prime}(a)=\hat{\phi}^{\prime}(b)=0$, for each positive $\epsilon$ we can find a $C^{\infty}$-function $\psi^{\prime}$ with support in $[a, b]$ such that

$$
-\hat{\phi}^{\prime}(t)<\psi^{\prime}(t)<-\hat{\phi}^{\prime}(t)+\epsilon
$$

for $t \in I_{i}$. Moreover, since

$$
0<\hat{\phi}\left(x_{i+1}\right)-\hat{\phi}\left(x_{i}\right)=\int_{x_{i}}^{x_{i+1}} \hat{\phi}^{\prime}(x) d x
$$

by choosing $\epsilon$ appropriately we can assume that

$$
\int_{a}^{b} \psi^{\prime}(t) d t=0
$$

If we set $\psi(t)=\int_{x_{i}}^{t} \psi^{\prime}(x) d x$, we see that $\phi=\hat{\phi}+\psi$ satisfies the interpolation conditions and is strictly increasing on $I_{i}$. This construction can clearly be applied to other intervals, as required, to obtain a $\phi \in C^{\infty}$ that satisfies the interpolation conditions and is strictly increasing on $\left[x_{1}, x_{M}\right]$.

Note that there are many ways to construct the change of parameter $\phi$. The interpolation conditions (15) only determine it partially; in many cases the unique polynomial of lowest degree that solves (15) will be an allowable change of parameter.

\section{A general approach to High-ACCURACy PARAMETRIC INTERPOLATion}

Interpolation by polynomials can conveniently be expressed through the Newton form and divided differences. Define the sequence $\left(t_{i}\right)_{i=1}^{m}$ by

$$
\left(t_{i}\right)_{i=1}^{m}=(\overbrace{x_{1}, \ldots, x_{1}}^{\ell_{1} \text { times }}, \ldots, \overbrace{x_{N}, \ldots, x_{N}}^{\ell_{N} \text { times }}),
$$

and define $\left(s_{i}\right)_{i=1}^{m}$ by $t_{i}=\phi\left(s_{i}\right)$ for a given allowable change of parameter $\phi$. From elementary numerical analysis we then know that the interpolating parametric polynomial curve $\boldsymbol{p}$ of degree $m-1$ which interpolates $\boldsymbol{f} \circ \phi$ at $\left(s_{i}\right)_{i=1}^{m}$ may be written as

$$
\begin{aligned}
\boldsymbol{p}(s)= & (\boldsymbol{f} \circ \phi)\left(s_{1}\right)+\left(s-s_{1}\right)\left[s_{1}, s_{2}\right](\boldsymbol{f} \circ \phi) \\
& +\cdots+\left(s-s_{1}\right) \cdots\left(s-s_{m-1}\right)\left[s_{1}, \ldots, s_{m}\right](\boldsymbol{f} \circ \phi) \\
= & \boldsymbol{p}_{1}(s)+\cdots+\boldsymbol{p}_{m-1}(s)
\end{aligned}
$$

where

$$
\boldsymbol{p}_{i}(s)=\left(s-s_{1}\right) \cdots\left(s-s_{i-1}\right)\left[s_{1}, \ldots, s_{i}\right](\boldsymbol{f} \circ \phi) .
$$

The error can be written as

$$
\boldsymbol{e}(s)=\left(s-s_{1}\right) \cdots\left(s-s_{m}\right)\left[s_{1}, \ldots, s_{m}, s\right](\boldsymbol{f} \circ \phi) .
$$

If we combine this with our concept of stability, see Definition 3, we obtain the following result, which formalizes the preliminary discussion in $\S 2$, cf. (8). 
Theorem 7. Let $\boldsymbol{f}$ be a parametric curve in $\mathbb{R}^{q}$, defined on the interval $[0, h]$, and suppose that there is a change of parameter $\phi_{h}$ with $\phi_{h}(0)=0$ and $\phi_{h}(h)=h$, and a polynomial $\boldsymbol{p}$ of degree $n \leq m-1$ that interpolates $\boldsymbol{f} \circ \phi$ at $m$ points $s_{1}, s_{2}, \ldots$, $s_{m}$ in $[0, h]$. Then

$$
d(\boldsymbol{p}, \boldsymbol{f}) \leq \sup _{s \in[0, h]}\left|\boldsymbol{p}(s)-\boldsymbol{f}\left(\phi_{h}(s)\right)\right| \leq h^{m} q \max _{1 \leq j \leq q} \max _{\theta \in(0, h)}\left|D^{m}\left(f_{j} \circ \phi_{h}\right)(\theta)\right| / m !,
$$

provided that $\boldsymbol{f} \in C^{m}$. The approximation order is $m$ if in addition the change of parameter $\phi_{h}$ is stable of order $m$ at 0.

Proof. The only claim that remains to be proved is the last inequality in (16). This follows by applying the well-known fact $\left[s_{1}, s_{2}, \ldots, s_{m}, s\right] g=g^{(m)}(\theta) / m$ ! (valid for sufficiently smooth $g$ ) to each component of $\boldsymbol{f} \circ \phi$. If $s \in[0, h]$, then $\theta \in(0, h)$. If $\phi_{h}$ is stable of order $m$, then the derivatives in (16) will remain bounded as $h$ tends to zero, so the approximation order will be $m$.

We are now in a position where we can explain our approach to parametric interpolation in more detail. If we consider $\phi$ to be unspecified, we recall from the previous section that each interpolation condition introduces an arbitrary parameter (the $\beta$ 's). In total we therefore have $m$ parameters which we may utilize to improve the approximation in one way or another. Note that a linear change of parametrization does not change the approximation in any essential way so that two of the free parameters are not of any interest for approximation purposes. This is in fact the reason why we may assume that $s_{1}=\phi\left(t_{1}\right)=t_{1}$ and $s_{m}=\phi\left(t_{m}\right)=t_{m}$. We are left with $m-2$ parameters to play with. In the $\beta$-spline paradigm [1], these are called shape parameters and are given to the curve designer as controls to obtain a desirable shape.

The alternative which we will pursue here is to choose the $\beta$ 's in such a way that the degree of $\boldsymbol{p}$ is reduced. This we do by requiring

$$
\left[s_{1}, \ldots, s_{m-i}\right](\boldsymbol{f} \circ \phi)=0,
$$

so that $\boldsymbol{p}_{m-i} \equiv 0$ for $i=0,1, \ldots, k$, with $k$ as large as possible. The number of such conditions that we can hope to enforce obviously depends on the dimension of the Euclidean space in which we are working. In the plane, each condition of type (17) introduces two scalar constraints. Since we have $m-2$ free $\beta$ 's, we can enforce at most $\lfloor(m-2) / 2\rfloor$ such conditions. This would reduce the degree of the interpolant to $n=m / 2$ for even $m$, and to $n=1+(m-1) / 2$ for odd $m$. If this can be carried through in a stable way, we still have the same approximation order $m$. In other words, we have a scheme that for polynomials of degree $n$ gives approximation order $2 n$. Note, however, that we have no guarantee that this can be done; the conditions (17) usually involve the $\beta$-parameters in a nonlinear way. We summarize this in a conjecture, see Rababah [18] for a similar conjecture.

Conjecture. Let $\boldsymbol{f} \in \mathbb{R}^{d}$ be a parametric curve. Then $\boldsymbol{f}$ can be interpolated at $m$ points by a polynomial of degree

$$
n_{0}=m-1-\left\lfloor\frac{m-2}{d}\right\rfloor .
$$


Equivalently, a polynomial of degree $n$ can be made to interpolate $\boldsymbol{f}$ at

$$
m_{0}=n+1+\left\lfloor\frac{n-1}{d-1}\right\rfloor
$$

points, yielding an approximation order of $m_{0}$ (the notation $\lfloor x\rfloor$ denotes the greatest integer not greater than $x$ ).

This conjecture is known to be true in some special cases, and we will extend these results below, but some conditions on the curve $f$ will be necessary.

As explained above, our schemes amount to choosing some reparametrization of $f$ which reduces the degree of an interpolating polynomial. The next result shows that the result is essentially independent of the initial parametrization of $\boldsymbol{f}$.

Proposition 8. Let $\boldsymbol{f}$ be a given parametric curve defined on the interval $I=$ $[0, h]$, and let $\boldsymbol{f}_{1}=\boldsymbol{f} \circ \psi_{1}$ and $\boldsymbol{f}_{2}=\boldsymbol{f} \circ \psi_{2}$ be two regular reparametrizations also defined on I. Let $\mathbb{P}_{n, m}^{i}$ denote the set of polynomial curves of degree $n$ that interpolate some reparametrization of $\boldsymbol{f}_{i}$ at $m$ points in $[0, h]$. Then $\mathbb{P}_{n, m}^{1}=\mathbb{P}_{n, m}^{2}$.

Proof. Let $\boldsymbol{p} \in \mathbb{P}_{n, m}^{1}$. Then $\boldsymbol{p}$ interpolates $\boldsymbol{f}_{1} \circ \phi_{1}$ for some reparametrization $\phi_{1}$. Now

$$
\boldsymbol{f}_{1} \circ \phi_{1}=\boldsymbol{f} \circ \psi_{1} \circ \phi_{1}=\boldsymbol{f} \circ \psi_{2} \circ \psi_{2}^{-1} \circ \psi_{1} \circ \phi_{1}=\boldsymbol{f}_{2} \circ \phi_{2},
$$

where $\phi_{2}=\psi_{2}^{-1} \circ \psi_{1} \circ \phi_{1}$. From this we conclude that $\boldsymbol{p}$ is also in $\mathbb{P}_{n, m}^{2}$. This argument is clearly symmetric, and hence $\mathbb{P}_{n, m}^{1}=\mathbb{P}_{n, m}^{2}$.

Proposition 8 gives us the freedom to work in whatever parametrization of $\boldsymbol{f}$ that is most convenient. We can therefore omit the superscript and write $\mathbb{P}_{n, m}$. Note, however, that $\mathbb{P}_{n, m}$ may contain many solution curves, or none.

We emphasize that the above conjecture involves two separate, but related problems. The first is whether the interpolant exists, the other whether the family of parameter changes $\phi_{h}$ in Theorem 7 , and therefore the interpolant, remains bounded as the interpolation points tend to a common limit. Both the question of existence and stability seem to be difficult to answer in general, but in the following we establish some results that will be useful in studying stability.

The most interesting case of the conjecture is when the floor function is exact, i.e., when $n-1=k(d-1)$ for some positive integer $k$. We then have the following "procedure" to determine the interpolating polynomial.

Procedure 9. Let $\boldsymbol{f}$ be a curve in $\mathbb{R}^{d}$ defined on the interval $[0, h]$, let $m$ be an integer of the form $m=k d+2$ with $k$ a positive integer, and let the integer $n$ be given by $n=k(d-1)+1$. If $t_{1}, t_{2}, \ldots, t_{m}$ are $m$ (not necessarily distinct) interpolation points in $[0, h]$, then a polynomial curve $\boldsymbol{p}$ of degree $n$ and a change of parameter $\phi$ such that $\left(\boldsymbol{p} \circ \phi^{-1}\right)\left(t_{i}\right)=\boldsymbol{f}\left(t_{i}\right)$ for $i=1,2, \ldots, m$ can be found by solving the system of $k d=m-2$ equations

$$
\left[s_{1}, \ldots, s_{i}\right](\boldsymbol{f} \circ \phi)=0 \quad \text { for } i=n+2, n+3, \ldots, m,
$$

where $s_{i}$ is given by $t_{i}=\phi\left(s_{i}\right)$ for $i=1,2, \ldots, m$.

Let us consider some of the interpolation schemes suggested by Procedure 9 . For $d=2$ (plane curves) we have $m=2 k+2$ and $n=k+1$ for some positive integer $k$, or $m=2 n$. The equations (20) can then be interpreted as a reduction of degree from $2 n-1$ to $n$. The simplest case is $k=1$, which corresponds to 
quadratic $(n=2)$ interpolation at $m=4$ points. Any cubic interpolation scheme for functions therefore becomes a quadratic scheme for planar curves.

The simplest scheme to analyze mathematically is the one where all four points are equal, which corresponds to the two curves $\boldsymbol{f}$ and $\boldsymbol{p}$ having fourth-order geometric contact at the point. We shall see in the next section that this is possible if the curvature is nonzero at the point. This Taylor scheme is obviously fourth-order accurate if it is solvable, since there is no variable change of parameter.

Since the equations that characterize the polynomial approximation depend smoothly on the interpolation points, quadratic schemes that interpolate four points are always solvable and fourth-order accurate in a neighborhood of a point with nonzero curvature (in this case the Jacobian is also nonsingular, cf. the next section). For interpolation points spaced further apart, the solvability question must be treated specially. One way of grouping the four points is by performing Hermite interpolation of position and tangent directions at two points. It is easy to see that this classical scheme is solvable provided the two tangent directions are linearly independent. As we approach a point with zero curvature, we may still have solvability, but the change of parameter is not stable and in the limit there is no solution. Another natural scheme is interpolation at four distinct points. Solvability of this scheme is treated in [13].

Consider next the case where $k=2$, so that we have $m=6$ interpolation points and the degree of the interpolant is $n=3$, corresponding to quintic schemes, where the degree is reduced by two. In $\S 6$ we show that the Taylor scheme in this case is always solvable unless the curvature of the curve has a double zero at the point. We also show that all cubic schemes are sixth-order accurate and solvable in a neighborhood of such points. As for quadratics, the solvability question must be treated separately when the points are spaced further apart. Maybe the most natural scheme is a two-point Hermite scheme where the interpolant has thirdorder contact with the curve at two points. This corresponds to interpolation of position, tangent direction and curvature at two points and has been treated in [2], see also [11]. Another natural cubic scheme is a three-point Hermite scheme which interpolates position and tangent direction at three points. Of course, there is also the possibility of interpolation at six distinct points.

As the value of $k$ increases, we get schemes with increasing accuracy (we gain two orders of accuracy for each increase in $k$ ), but the determining equations of the schemes also become increasingly difficult, both to solve and to analyze.

In space $(d=3)$, the simplest schemes correspond to interpolation at $m=5$ points by cubic $(n=3)$ curves, which are degenerate quartic curves. In the next section we show that the Taylor scheme is always solvable provided the torsion is nonzero at the point. Because the Jacobian of the equations is nonsingular at such a point, all cubic schemes in space are fifth-order accurate and solvable in a neighborhood of the point. If we separate the points, a natural configuration is interpolation of position and tangent at two points and position at one point in between. Increasing $k$ to two, we come to quintic curves $(n=5)$ interpolating at $m=8$ points. For such schemes we have no results.

In arbitrary dimension $d$, we show that the simplest schemes $(k=1)$ with $m=d+2$ and $n=d$ are always solvable in the Taylor case provided the highest curvature is nonzero. By studying the Jacobian, we find that general schemes of this type are always solvable and $(d+2)$-order accurate in a neighborhood of such points. 


\section{General SOlvability And Stability Results}

This section is devoted to proving some results about the set of equations (20). From the previous section we know that the interpolation conditions lead to conditions on the value of $\phi$ and its derivatives at the distinct points among $s_{1}, \ldots, s_{m}$, see (12). In fact, since we have $m$ conditions, we can enforce all the conditions if we assume that $\phi$ is a polynomial of degree $m-1$. A convenient way to represent $\phi$ is therefore

$$
\begin{gathered}
\phi(s)=\alpha_{1} s+\alpha_{2} s^{2} / 2+\cdots+\alpha_{m-1} s^{m-1} /(m-1) ! \\
\alpha_{1}=1-\alpha_{2} h / 2-\cdots-\alpha_{m-1} h^{m-2} /(m-1) ! .
\end{gathered}
$$

Here, the conditions $\phi(0)=0$ and $\phi(h)=h$ have been incorporated, i.e., we have assumed that $s_{1}=t_{1}=0$ and $s_{m}=t_{m}=h$. We see that $\phi$ now depends on $m-2=k d$ free parameters $\left(\alpha_{i}\right)_{i=2}^{m-1}$, see Procedure 9 , which is the minimal number in order to satisfy the $m-2$ constraints in (20).

Having parametrized $\phi$, we can define the real functions $\boldsymbol{\Phi}_{i, m}=\boldsymbol{\Phi}_{i}: \mathbb{R}^{2 m-2} \mapsto \mathbb{R}$ by

$$
\boldsymbol{\Phi}_{i}\left(\alpha_{2}, \ldots, \alpha_{m-1} ; s_{1}, \ldots, s_{m}\right)=\left[s_{1}, \ldots, s_{i}\right](\boldsymbol{f} \circ \phi) \quad \text { for } 1 \leq i \leq m .
$$

The system (20) can then be expressed as

$$
\boldsymbol{\Phi}_{i}\left(\alpha_{2}, \ldots, \alpha_{m-1} ; s_{1}, \ldots, s_{m}\right)=0 \quad \text { for } i=n+2, n+3, \ldots, m .
$$

Note that we ought to let $\boldsymbol{\Phi}_{i}$ depend on the $t_{i}$ (which are typically given numbers) and not the $s_{i}$ (which we do not know until the parametrization $\phi$ is known), but since we always assume $\phi$ to be an allowable change of parameter and therefore invertible, we can always make the change of variable $t_{i}=\phi\left(s_{i}\right)$.

The following lemma is fundamental in what follows.

Lemma 10. If $\boldsymbol{f}$ is smooth, the function $\boldsymbol{\Phi}_{i}$ is smooth, and the partial derivative $D_{j}$ with respect to $\alpha_{j}$ is given by

$$
D_{j} \boldsymbol{\Phi}_{i}\left(\alpha_{2}, \ldots, \alpha_{m-1} ; s_{1}, \ldots, s_{i}\right)=\left[s_{1}, \ldots, s_{i}\right]\left(r_{j} \cdot \boldsymbol{f}^{\prime} \circ \phi\right),
$$

where the function $r_{j}$ is given by $r_{j}(s)=\left(s^{j}-h^{j-1}\right) / j$ ! (the $\cdot$ denotes multiplication so that the function $r_{i} \cdot \boldsymbol{f}^{\prime} \circ \phi$ has the value $r_{j}(s) \boldsymbol{f}^{\prime}(\phi(s))$ at $\left.s\right)$. This formula is also valid for $h=0$.

Proof. It is well known that a divided difference depends smoothly on its arguments provided $\boldsymbol{f}$ is sufficiently smooth. The derivative of $\boldsymbol{\Phi}_{i}$ with respect to $\alpha_{j}$ is easily obtained by, for example, taking limits.

From Lemma 10 it is easy to compute the Jacobian matrix of the system of equations (22) and to give conditions for nonsingularity.

Lemma 11. Let $\boldsymbol{\Psi}_{i}: \mathbb{R}^{m-2} \mapsto \mathbb{R}^{m-2}$ denote the restriction of $\boldsymbol{\Phi}_{i}$ in (22) to the first $m-2=k d$ variables $\left(\alpha_{i}\right)_{i=2}^{m-1}$. The mapping $\boldsymbol{\Psi}: \mathbb{R}^{m-2} \mapsto \mathbb{R}^{m-2}$ given by $\mathbf{\Psi}=\left(\mathbf{\Psi}_{m}, \mathbf{\Psi}_{m-1}, \ldots, \mathbf{\Psi}_{n+2}\right)$ has the Jacobian matrix $J$ with rows $d(i-1)+1, \ldots$, di given by

$$
\left(\left[s_{1}, \ldots, s_{m-i+1}\right]\left(r_{j+1} \cdot \boldsymbol{f} \circ \phi\right)\right)_{j=1}^{m-2},
$$

for $i=1, \ldots, k$. This matrix is nonsingular if and only if the only polynomial $r$ of the form $r(t)=\sum_{i=2}^{m-1} c_{i} r_{i}(t)$ that solves the set of equations

$$
\left[s_{1}, \ldots, s_{m-i+1}\right]\left(r \cdot \boldsymbol{f}^{\prime} \circ \phi\right)=0 \quad \text { for } i=1,2, \ldots, k,
$$

is the polynomial $r(t) \equiv 0$. 
From the implicit function theorem we now have the following proposition.

Proposition 12. Suppose that the system (22) has a solution $\boldsymbol{\alpha}^{*}$ for a given set of interpolation points $\boldsymbol{s}^{*}$. If the Jacobian matrix in Lemma 11 with respect to $\boldsymbol{\alpha}$ is nonsingular at $\left(\boldsymbol{\alpha}^{*}, \boldsymbol{s}^{*}\right)$, then there is some neighborhood $U$ of $\boldsymbol{s}^{*}$ where the set of equations can be solved for $\boldsymbol{\alpha}$ in terms of $\boldsymbol{s}$.

This has immediate consequences for stability (cf. Definition 3).

Corollary 13. The system of equations (22) yields a stable change of parameter $\phi$ of order $m-1$ as $h$ tends to zero, if the Jacobian matrix of (22) with respect to $\boldsymbol{\alpha}$ at the point $s_{1}=s_{2}=\cdots=s_{m}=0$ is nonsingular, and the system is solvable at this point.

Proof. Under the conditions of the corollary the $\alpha$ 's will depend smoothly on the $s_{i}$. But note that $\alpha_{i}=\phi^{(i)}(0)$, so that $\phi$ will also depend smoothly on the $s_{i}$.

The problem of stability has now been reduced to the problem of showing that the Jacobian matrix of (22) is nonsingular when all the interpolation points coalesce. In addition, we also need to know that the corresponding interpolation problem has a solution. For the simplest nontrivial schemes in each space dimension these problems are settled by the following theorem.

Theorem 14. Let $\boldsymbol{f}$ be a curve in $\mathbb{R}^{d}$. If the first $d$ derivatives at $s=0$ span $\mathbb{R}^{d}$, i.e., if $\operatorname{span}\left\{\boldsymbol{f}^{\prime}(0), \ldots, \boldsymbol{f}^{(d)}(0)\right\}=\mathbb{R}^{d}$, there is a unique polynomial curve $\boldsymbol{p}$ of degree $d$ which agrees with $\boldsymbol{f}$ with geometric continuity of order $d+1$ at $s=0$, yielding an approximation order of $m=d+2$ in a neighborhood of $s=0$. Moreover, there is a neighborhood $U$ of $0 \in \mathbb{R}^{d+2}$ such that for each set of $d+2$ interpolation points $\boldsymbol{s}=\left(s_{1}, \ldots, s_{d+2}\right)$ in $U$, there is a polynomial of degree $d$ which interpolates $\boldsymbol{f}$ at the $d+2$ points of $\boldsymbol{s}$. As the interpolation points tend to 0 , the corresponding interpolant is $d+2$-order accurate.

Proof. Consider first solvability of the problem in the case where all the interpolation points are equal. The equations (22) then reduce to one vector equation of dimension $d$, namely $D^{d+1}(\boldsymbol{f} \circ \phi)(0)=0$, with $h=0$ or $\alpha_{1}=1$ in (21). From Lemma 5 we know that this is equivalent to

$$
\sum_{j=1}^{d+1} a_{d+1, j} \boldsymbol{f}^{(j)}(0)=0
$$

with $a_{d+1, j}$ given by

$$
a_{d+1, j}=\sum_{\substack{i_{1}+i_{2}+\cdots+i_{j}=d+1 \\
i_{k}>0, k=1, \ldots, j}}\left[\begin{array}{c}
d+1 \\
i_{1}, i_{2}, \ldots, i_{j}
\end{array}\right] \alpha_{i_{1}} \alpha_{i_{2}} \cdots \alpha_{i_{j}},
$$

where we now have $\alpha_{1}=1$. If we start from the end of the sum in (23), we see from (24) that $a_{d+1, d+1}=\alpha_{1}^{d+1}=1$. Equation (23) can therefore be rewritten as

$$
\sum_{j=1}^{d} a_{d+1, j} \boldsymbol{f}^{(j)}(0)=-\boldsymbol{f}^{(d+1)}(0) .
$$


Since $a_{d+1, d}$ requires a sum of length $d$ in (24), we see that $a_{d+1, d}=c_{d} \alpha_{1}^{d-1} \alpha_{2}=$ $c_{d} \alpha_{2}$ for some nonzero constant $c_{d}$. If we let $\left\langle\boldsymbol{v}_{1}, \ldots, \boldsymbol{v}_{d}\right\rangle$ denote the determinant of the $d \times d$-matrix with rows $\left(\boldsymbol{v}_{i}\right)_{i=1}^{d}$, we therefore have

$$
\alpha_{2}=-\frac{\left\langle\boldsymbol{f}^{\prime}(0), \boldsymbol{f}^{\prime \prime}(0), \ldots, \boldsymbol{f}^{(d-1)}(0), \boldsymbol{f}^{(d+1)}(0)\right\rangle}{c_{d}\left\langle\boldsymbol{f}^{\prime}(0), \boldsymbol{f}^{\prime \prime}(0), \ldots, \boldsymbol{f}^{(d)}(0)\right\rangle},
$$

provided the denominator is not zero, i.e., provided the first $d$ derivatives span $\mathbb{R}^{d}$. In the rest of the proof, we will use the abbreviation $\kappa_{d-1}=\left\langle\boldsymbol{f}^{\prime}(0), \boldsymbol{f}^{\prime \prime}(0), \ldots\right.$, $\left.\boldsymbol{f}^{(d)}(0)\right\rangle$.

Proceeding, we find that $a_{d+1, d-1}=c_{d-1,1} \alpha_{3} \alpha_{1}^{d-2}+c_{d-1,2} \alpha_{2}^{2} \alpha_{1}^{d-1}$ for suitable constants $c_{d-1,1}$ and $c_{d-1,2}$. Since $\alpha_{2}$ is now known, we can solve for $\alpha_{3}$ as we did for $\alpha_{2}$ provided $\kappa_{d-1} \neq 0$. In general, we see that in the sum defining $a_{d+1, d-k}$ there will only be one term that involves only $\alpha_{k+2}$ and $\alpha_{1}$, since there is only one way to write $d+1$ as a sum of $d-k$ positive integers with one of them equal to $k+2$, namely as

$$
d+1=\underbrace{(k+2)+\overbrace{1+1+\cdots+1}^{d-k-1 \text { times }}}_{d-k \text { terms }} .
$$

We can therefore determine $\alpha_{k+2}$, as above, in terms of the already known $\alpha$ 's, provided $\kappa_{d-1} \neq 0$. The solution is clearly unique. This completes the proof of solvability.

For the stability, we use Corollary 13 and consider the $d \times d$ Jacobian matrix

$$
J=\left[D^{d+1}\left(r_{2} \cdot \boldsymbol{f}^{\prime} \circ \phi\right)(0), \ldots, D^{d+1}\left(r_{d+1} \cdot \boldsymbol{f}^{\prime} \circ \phi\right)(0)\right],
$$

where $r_{i}(t)=t^{i} / i$ ! (remember that $h=0$ in the formula in Lemma 10). We differentiate the products using Leibniz's rule and find

$$
\begin{aligned}
& J=\left[b_{d+1,2} D^{(d-1)}\left(\boldsymbol{f}^{\prime} \circ \phi\right)(0),\right. \\
& \left.\quad b_{d+1,3} D^{(d-2)}\left(\boldsymbol{f}^{\prime} \circ \phi\right)(0), \ldots, b_{d+1, d} D\left(\boldsymbol{f}^{\prime} \circ \phi\right)(0),\left(\boldsymbol{f}^{\prime} \circ \phi\right)(0)\right],
\end{aligned}
$$

where $b_{d+1, i}=\left(\begin{array}{c}d+1 \\ i\end{array}\right)$. If we now consider the determinant of $J$, we see that column $d-1$ will be a linear combination of $\boldsymbol{f}^{\prime}(0)$ and $\boldsymbol{f}^{\prime \prime}(0)$. Since we already have the vector $\boldsymbol{f}^{\prime}(0)$ in column, the determinant does not change if we remove it from column $d-1$. In general, column $d-k$ will be a linear combination of the first $k+1$ derivatives of $\boldsymbol{f}$, but the first $k$ derivatives already occur in columns $d-k+1, \ldots, d$ and hence do not contribute to the determinant. Note also that the coefficient multiplying $\boldsymbol{f}^{(k+1)}(0)$ is $\alpha_{1}^{k}=1$. We therefore conclude that

$$
\operatorname{det} J=b_{d+1,2} b_{d+1,3} \cdots b_{d+1, d}\left\langle\boldsymbol{f}^{(d)}(0), \ldots, \boldsymbol{f}^{\prime}(0)\right\rangle \text {. }
$$

In other words, the determinant is nonzero if $\kappa_{d-1} \neq 0$. The result now follows from Corollary 13.

Note that the determinant $\kappa_{d-1}(0)$ is closely related to the highest $(d-1)$ st curvature of $\boldsymbol{f}$ at 0 , in that the determinant is zero if the highest curvature is zero, see [10] and [24]. In the plane the highest curvature is the ordinary curvature,

$$
\kappa=\frac{\left\langle\boldsymbol{f}^{\prime}(0), \boldsymbol{f}^{\prime \prime}(0)\right\rangle}{\left|\boldsymbol{f}^{\prime}(0)\right|^{3}},
$$


while in space the highest curvature is the torsion defined by

$$
\tau=\frac{\left\langle\boldsymbol{f}^{\prime}(0), \boldsymbol{f}^{\prime \prime}(0), \boldsymbol{f}^{\prime \prime \prime}(0)\right\rangle}{\left|\boldsymbol{f}^{\prime}(0) \times \boldsymbol{f}^{\prime \prime}(0)\right|^{2}}
$$

This gives the following corollary.

Corollary 15. In the plane, quadratic interpolation to four points is always possible in a neighborhood of a point where the curvature is nonzero, and all such schemes are fourth-order accurate. In space, cubic interpolation at five points is always possible in the neighborhood of a point where the torsion is nonzero, and all such schemes are fifth-order accurate.

As an example of these ideas, consider interpolation of four coalescing points in the plane with quadratics in some more detail. This scheme will also be important in our analysis of cubic schemes in the next section.

Example. Quadratic Taylor approximation in the plane. If the parametric curve is $\boldsymbol{f}$, and $\boldsymbol{f} \circ \phi$ is some reparametrization, we can approximate $\boldsymbol{f}$ at $t=0$ by Taylor expansions of $\boldsymbol{f} \circ \phi$, where we may assume that $\phi(0)=0$. The quadratic Taylor approximation looks like

$$
\boldsymbol{f}(\phi(s))=\boldsymbol{f}(0)+\alpha_{1} \boldsymbol{f}^{\prime}(0) s+\left(\alpha_{2} \boldsymbol{f}^{\prime}(0)+\alpha_{1}^{2} \boldsymbol{f}^{\prime \prime}(0)\right) \frac{s^{2}}{2},
$$

where $\alpha_{1}=\phi^{\prime}(0)$ and $\alpha_{2}=\phi^{\prime \prime}(0)$. From this, we see, as expected, that there is no loss in choosing $\alpha_{1}=1$ (replace the parameter $s$ by $s / \alpha_{1}$ ). We also see that it is in general impossible to reduce a quadratic approximation to a linear one by choosing $\alpha_{2}$ in a clever way.

However, it is clear that the cubic Taylor approximation to $\boldsymbol{f} \circ \phi$ reduces to a quadratic if $D^{3}(\boldsymbol{f} \circ \phi)(0)=0$ (see $\left.(17)\right)$, i.e., if

$$
\alpha_{3} \boldsymbol{f}^{\prime}(0)+3 \alpha_{2} \boldsymbol{f}^{\prime \prime}(0)+\boldsymbol{f}^{\prime \prime \prime}(0)=0
$$

where we have assumed that $\alpha_{1}=\phi^{\prime}(0)=1$. Employing the bracket notation used in the proof of Theorem 14, we find

$$
\alpha_{2}=-\frac{\left\langle\boldsymbol{f}^{\prime}(0), \boldsymbol{f}^{\prime \prime \prime}(0)\right\rangle}{3\left\langle\boldsymbol{f}^{\prime}(0), \boldsymbol{f}^{\prime \prime}(0)\right\rangle} \quad \text { and } \quad \alpha_{3}=\frac{\left\langle\boldsymbol{f}^{\prime \prime}(0), \boldsymbol{f}^{\prime \prime \prime}(0)\right\rangle}{\left\langle\boldsymbol{f}^{\prime}(0), \boldsymbol{f}^{\prime \prime}(0)\right\rangle} .
$$

Since the curvature of $\boldsymbol{f}$ at $t=0$ is given by $\kappa(0)=\left\langle\boldsymbol{f}^{\prime}(0), \boldsymbol{f}^{\prime \prime}(0)\right\rangle /\left|\boldsymbol{f}^{\prime}(0)\right|^{3}$, this scheme is only defined when $\kappa(0) \neq 0$, in accordance with Theorem 14 .

Note that as an extra bonus from determining the quadratic Taylor approximation we have found a reparametrization $\boldsymbol{g}=\boldsymbol{f} \circ \phi$ of $\boldsymbol{f}$ with the property that $\boldsymbol{g}^{\prime \prime \prime}(0)=0$. This will be useful in the next section.

\section{Stability of CUbic Schemes in the Plane}

In the previous section we developed some results about the stability of the parametric interpolation procedure valid in any space dimension. The cubic case in the plane is an important case that is not covered by these results. To study this in detail, we start by considering the Taylor case. In what follows some abbreviations will be useful. The notation $\boldsymbol{f}^{\prime}$ will denote $\boldsymbol{f}^{\prime}(0)$, as above the bracket notation $\langle\boldsymbol{a}, \boldsymbol{b}\rangle$ will denote the determinant of the matrix with rows $\boldsymbol{a}$ and $\boldsymbol{b}$, while $d_{i, j}=$ $\left\langle\boldsymbol{f}^{(i)}, \boldsymbol{f}^{(j)}\right\rangle$, the determinant of the $i$ th and $j$ th derivative of $\boldsymbol{f}$ at 0 . 
The cubic Taylor approximation to $\boldsymbol{f} \circ \phi$ will be sixth-order accurate at $t=0$ if $D^{4}(\boldsymbol{f} \circ \phi)(0)=D^{5}(\boldsymbol{f} \circ \phi)(0)=0$, that is, if

$$
\begin{array}{r}
\alpha_{4} \boldsymbol{f}^{\prime}+\left(4 \alpha_{3}+3 \alpha_{2}^{2}\right) \boldsymbol{f}^{\prime \prime}+6 \alpha_{2} \boldsymbol{f}^{\prime \prime \prime}+\boldsymbol{f}^{(4)}=0, \\
\alpha_{5} \boldsymbol{f}^{\prime}+\left(10 \alpha_{2} \alpha_{3}+5 \alpha_{4}\right) \boldsymbol{f}^{\prime \prime}+\left(15 \alpha_{2}^{2}+10 \alpha_{3}\right) \boldsymbol{f}^{\prime \prime \prime}+10 \alpha_{2} \boldsymbol{f}^{(4)}+\boldsymbol{f}^{(5)}=0,
\end{array}
$$

(recall that $\alpha_{1}=\phi^{\prime}(0)=1$ in the Taylor case). The following theorem shows that this system of equations always has a solution as long as the three vectors $\boldsymbol{f}^{\prime}, \boldsymbol{f}^{\prime \prime}$ and $\boldsymbol{f}^{\prime \prime \prime}$ span $\mathbb{R}^{2}$.

Theorem 16. Suppose that $\boldsymbol{f}$ is a curve with the property that $\operatorname{span}\left\{\boldsymbol{f}^{\prime}, \boldsymbol{f}^{\prime \prime}, \boldsymbol{f}^{\prime \prime \prime}\right\}=$ $\mathbb{R}^{2}$ at $t=0$. Then the system of equations (26)-(27) has at least one solution, so there is a cubic polynomial curve that is sixth-order accurate in a neighborhood of the point $t=0$. If the first three derivatives are linearly dependent, the system has no solution unless the first five derivatives are dependent, in which case $\boldsymbol{f}$ agrees with a straight line up to sixth-order.

Proof. Suppose first that $\left\langle\boldsymbol{f}^{\prime}, \boldsymbol{f}^{\prime \prime}\right\rangle \neq 0$. Then we can form four scalar equations from (26) and (27) by taking determinants with $\boldsymbol{f}^{\prime}$ and $\boldsymbol{f}^{\prime \prime}$,

$$
\begin{aligned}
\alpha_{4} d_{1,2}-6 \alpha_{2} d_{2,3}-d_{2,4} & =0 \\
\left(4 \alpha_{3}+3 \alpha_{2}^{2}\right) d_{1,2}+6 \alpha_{2} d_{1,3}+d_{1,4} & =0 \\
\alpha_{5} d_{1,2}-\left(15 \alpha_{2}^{2}+10 \alpha_{3}\right) d_{2,3}-10 \alpha_{2} d_{2,4}-d_{2,5} & =0 \\
\left(10 \alpha_{2} \alpha_{3}+5 \alpha_{4}\right) d_{1,2}+\left(15 \alpha_{2}^{2}+10 \alpha_{3}\right) d_{1,3}+10 \alpha_{2} d_{1,4}+d_{1,5} & =0
\end{aligned}
$$

We see that $\alpha_{4}, \alpha_{3}$ and $\alpha_{5}$ can be found in terms of $\alpha_{2}$ from (28), (29) and (30), respectively. If these expressions are substituted in (31), we obtain a cubic equation in $\alpha_{2}$ with coefficients that are polynomials in the $d_{i, j}$. In particular, the coefficient multiplying $\alpha_{2}^{3}$ is $d_{1,2}^{2}$. Since we have assumed that $d_{1,2} \neq 0$, this equation, therefore, always has at least one real solution. The other unknowns can then be found from $\alpha_{2}$.

Suppose next that $d_{1,2}=0$, but $d_{1,3} \neq 0$. Then we take determinants in $(26)$ and (27) with $\boldsymbol{f}^{\prime}$ and $\boldsymbol{f}^{\prime \prime \prime}$ and find

$$
\begin{aligned}
6 \alpha_{2} d_{1,3}+d_{1,4} & =0 \\
\alpha_{4} d_{1,3}+\left(4 \alpha_{3}+3 \alpha_{2}^{2}\right) d_{2,3}-d_{3,4} & =0 \\
\left(15 \alpha_{2}^{2}+10 \alpha_{3}\right) d_{1,3}+10 \alpha_{2} d_{1,4}+d_{1,5} & =0 \\
\alpha_{5} d_{1,3}+\left(10 \alpha_{2} \alpha_{3}+5 \alpha_{4}\right) d_{2,3}-10 \alpha_{2} d_{3,4}+d_{3,5} & =0
\end{aligned}
$$

We see that we can find $\alpha_{2}$ from (32), then $\alpha_{3}$ from (34), then $\alpha_{4}$ from (33) and finally $\alpha_{5}$ from (35), all by solving a linear equation, provided $d_{1,3} \neq 0$.

If the first three derivatives are linearly dependent, i.e., if $d_{1,2}=d_{1,3}=d_{2,3}=0$, it is easy to see that the first five derivatives must be dependent for a solution to exist. If this is the case, we have infinitely many solutions.

Theorem 16 only tells us that six-fold interpolation at $t=0$ is possible, but the results of the previous section tell us that if the Jacobian of the system of equations is nonzero at a solution, then there is also a cubic polynomial curve 
that interpolates $\boldsymbol{f}$ at six points in any neighborhood of $t=0$. Unfortunately, the condition $\operatorname{span}\left\{\boldsymbol{f}^{\prime}, \boldsymbol{f}^{\prime \prime}, \boldsymbol{f}^{\prime \prime \prime}\right\}=\mathbb{R}^{2}$ is not sufficient to ensure that the Jacobian is nonzero, but it turns out that even if the Jacobian is zero, interpolation at six points in a neighborhood of $t=0$ is possible.

Theorem 17. Suppose that $\boldsymbol{f}$ is a curve such that $\operatorname{span}\left\{\boldsymbol{f}^{\prime}(0), \boldsymbol{f}^{\prime \prime}(0), \boldsymbol{f}^{\prime \prime \prime}(0)\right\}=$ $\mathbb{R}^{2}$. If $|h|$ is sufficiently small, there is at least one cubic parametric curve that interpolates $\boldsymbol{f}$ at the six points $0, t_{1}, t_{2}, t_{3}, t_{4}, h$, where $0 \leq\left|t_{i}\right| \leq|h|$ for $1 \leq i \leq 4$. Any cubic approximation scheme that interpolates $\boldsymbol{f}$ in this way is therefore sixthorder accurate.

Proof. From Theorem 16 we know that the result is true for $h=0$; we just have to show that it is also true when the six interpolation points are in some neighborhood of $t=0$. If the Jacobian determinant of the system of equations is nonzero at the Taylor solution, we know from Proposition 12 that the equations are also solvable for six arbitrary interpolation points in a neighborhood of $t=0$.

From Lemma 11 we find that the Jacobian of the system of equations with all interpolation points at zero is

$$
\operatorname{det} J=\operatorname{det}\left[\begin{array}{cccc}
6\left(\alpha_{2} \boldsymbol{f}^{\prime \prime}+\boldsymbol{f}^{\prime \prime \prime}\right) & 4 \boldsymbol{f}^{\prime \prime} & \boldsymbol{f}^{\prime} & 0 \\
10\left(\alpha_{3} \boldsymbol{f}^{\prime \prime}+3 \alpha_{2} \boldsymbol{f}^{\prime \prime \prime}+\boldsymbol{f}^{(4)}\right) & 10\left(\alpha_{2} \boldsymbol{f}^{\prime \prime}+\boldsymbol{f}^{\prime \prime \prime}\right) & 5 \boldsymbol{f}^{\prime \prime} & \boldsymbol{f}^{\prime}
\end{array}\right] .
$$

Suppose first that $d_{1,2}=0$, so that $\left\langle\boldsymbol{f}^{\prime}, \boldsymbol{f}^{\prime \prime}\right\rangle=0$ but $d_{1,3}=\left\langle\boldsymbol{f}^{\prime}, \boldsymbol{f}^{\prime \prime \prime}\right\rangle$ is nonzero. Expanding the determinant, we find $J=-60 d_{1,3}^{2}$, and hence there is a solution for any set of six interpolation points in some neighborhood of $t=0$, and this solution depends smoothly on the interpolation points.

The case $d_{1,2}=d_{1,3}=0$ is trivial, as above in Theorem 16, so we assume now that $d_{1,2} \neq 0$. We can then reparametrize $\boldsymbol{f}$ so that $\boldsymbol{f}^{\prime \prime \prime}=0$ by using the change of parameter that is induced by the quadratic Taylor scheme, see the end of $\S 5$. In this case the equations (28)-(31) become

$$
\begin{aligned}
\alpha_{4} d_{1,2}-d_{2,4} & =0 \\
\alpha_{5} d_{1,2}-10 \alpha_{2} d_{2,4}-d_{2,5} & =0 \\
\left(4 \alpha_{3}+3 \alpha_{2}^{2}\right) d_{1,2}+d_{1,4} & =0 \\
\left(10 \alpha_{2} \alpha_{3}+5 \alpha_{4}\right) d_{1,2}+10 \alpha_{2} d_{1,4}+d_{1,5} & =0
\end{aligned}
$$

Now we subtract five times the first equation from the last, thereby eliminating $\alpha_{4}$. The determinant $\operatorname{det} J^{*}$ of the Jacobian $J^{*}$ of this system is not changed by this operation. For the unknowns $\alpha_{2}$ and $\alpha_{3}$ we obtain the reduced system

$$
\begin{aligned}
f_{1}=\left(4 \alpha_{3}+3 \alpha_{2}^{2}\right) d_{1,2}+d_{1,4} & =0 \\
f_{2}=10 \alpha_{2} \alpha_{3} d_{1,2}+10 \alpha_{2} d_{1,4}+d_{1,5}+5 d_{2,4} & =0 .
\end{aligned}
$$

Since the remaining two equations are linear in the only unknowns $\alpha_{4}$ and $\alpha_{5}$, we see that

$$
\operatorname{det} J^{*}=d_{1,2}^{2} \operatorname{det} J_{f}
$$


where $J_{f}$ is the Jacobian of the reduced system. If we now subtract $5 \alpha_{2}$ times $f_{1}$ from $2 f_{2}$, we obtain the new system

$$
\begin{aligned}
g_{1}=f_{1}=\left(4 \alpha_{3}+3 \alpha_{2}^{2}\right) d_{1,2}+d_{1,4} & =0, \\
g_{2}=2 f_{2}-5 \alpha_{2} f_{1}=p\left(\alpha_{2}\right)=-15 \alpha_{2}^{3} d_{1,2}+15 \alpha_{2} d_{1,4}+10 d_{2,4}+2 d_{1,5} & =0 .
\end{aligned}
$$

The Jacobian $J_{g}$ of this system satisfies $\operatorname{det} J_{g}=-d_{1,2} p^{\prime}\left(\alpha_{2}\right)$. By the chain rule, we therefore have

$$
\operatorname{det} J^{*}=2 d_{1,2}^{2} \operatorname{det} J_{g}=8 d_{1,2}^{3} p^{\prime}\left(\alpha_{2}\right),
$$

at a solution of the equations. Since $p$ is a cubic polynomial we can always find a solution for which $p^{\prime}\left(\alpha_{2}\right)$ is nonzero except when $p$ has one real root of multiplicity three. From (37) we see that this can only happen when

$$
\begin{aligned}
d_{1,4} & =0, \\
5 d_{2,4}+d_{1,5} & =0,
\end{aligned}
$$

and the root is $\alpha_{2}=0$. Since then the Jacobian is zero, we have to work harder to prove the theorem in this case, but it follows from the following sequence of lemmas.

The idea of the proof in the singular case (38) is via perturbation of the equations (28)-(31). We write this system in the form

$$
\boldsymbol{T}(\boldsymbol{\alpha})-\boldsymbol{d}=0,
$$

where

$$
\boldsymbol{d}=\left(d_{2,4},-d_{1,4}, d_{2,5},-d_{1,5}\right)
$$

and $\boldsymbol{T}(\boldsymbol{\alpha})$ denotes the remaining part of the system.

The norm that is being used in this section is the vector max-norm, which for a vector $\boldsymbol{x}=\left(x_{1}, \ldots, x_{m}\right)$ is defined by $\|\boldsymbol{x}\|=\max _{i}\left|x_{i}\right|$. Note that in what follows we always assume that $\boldsymbol{f}$ is parametrized so that $\boldsymbol{f}^{\prime \prime \prime}(0)=0$. We start by a lemma which shows that general parametric interpolation can be considered to be a perturbation of parametric Taylor interpolation. Without loss of generality we assume for the rest of this section that $h \geq 0$.

Lemma 18. Let $\boldsymbol{d}$ denote the column vector $\boldsymbol{d}=\left(d_{2,4},-d_{1,4}, d_{2,5},-d_{1,5}\right)^{T}$, and let $\boldsymbol{T}: \mathbb{R}^{4} \mapsto \mathbb{R}^{4}$ denote the nonlinear mapping so that

$$
\boldsymbol{T}(\boldsymbol{\alpha})-\boldsymbol{d}=0
$$

corresponds to the system of equations (28)-(31). Then the general system of equations

$$
\begin{aligned}
{\left[0, s_{1}, s_{2}, s_{3}, s_{4}\right](f \circ \phi) } & =0, \\
{\left[0, s_{1}, s_{2}, s_{3}, s_{4}, h\right](f \circ \phi) } & =0
\end{aligned}
$$

can be written

$$
\boldsymbol{T}(\boldsymbol{\alpha})+h \boldsymbol{s}_{h}(\boldsymbol{\alpha})=\boldsymbol{d},
$$


where it is assumed that $\phi$ is of the form (21), and $\boldsymbol{\alpha}=\left(\alpha_{2}, \alpha_{3}, \alpha_{4}, \alpha_{5}\right)$. For any $\boldsymbol{\beta}$ and $\boldsymbol{\gamma}$ in the ball $B(w)=\{\boldsymbol{x} \mid\|\boldsymbol{x}\| \leq w\}$ and any $h$ smaller than some $H$, the mapping $\boldsymbol{s}_{h}$ satisfies

$$
\left\|\boldsymbol{s}_{h}(\boldsymbol{\gamma})-\boldsymbol{s}_{h}(\boldsymbol{\beta})\right\| \leq C_{1}\|\boldsymbol{\gamma}-\boldsymbol{\beta}\| .
$$

Here, the symbol $C_{1}$ denotes a bounded constant that depends on the derivatives of $\boldsymbol{f}$ in a neighborhood of $[0, h]$ and the constants $w$ and $H$.

Proof. Equation (43) has four components, two from each of the vector equations (41) and (42). Since these two equations are similar, we only consider the first one in detail.

Recall that by Peano's representation theorem for divided differences we have

$$
\boldsymbol{\delta}_{4}(\boldsymbol{f})=4 !\left[0, s_{1}, s_{2}, s_{3}, s_{4}\right] \boldsymbol{f} \circ \phi=\int_{0}^{s_{4}} M_{4}(s)(\boldsymbol{f} \circ \phi)^{(4)}(s) d s,
$$

where $M_{4}(s)$ is the cubic B-spline with knots at $\left(0, s_{1}, s_{2}, s_{3}, s_{4}\right)$, normalized to have unit integral. Write $\boldsymbol{\delta}_{4}(\boldsymbol{f})$ as

$$
\begin{aligned}
\boldsymbol{\delta}_{4}(\boldsymbol{f}) & =(\boldsymbol{f} \circ \phi)^{(4)}(0)+\int_{0}^{s_{4}} M_{4}(s)\left[(\boldsymbol{f} \circ \phi)^{(4)}(s)-(\boldsymbol{f} \circ \phi)^{(4)}(0)\right] d s \\
& =(\boldsymbol{f} \circ \phi)^{(4)}(0)+h \boldsymbol{R}_{h}(\boldsymbol{\alpha}),
\end{aligned}
$$

where

$$
\boldsymbol{R}_{h}(\boldsymbol{\alpha})=(1 / h) \int_{0}^{s_{4}} M_{4}(s) \int_{0}^{s}\left(\boldsymbol{f} \circ \phi_{\boldsymbol{\alpha}}\right)^{(5)}(u) d u d s
$$

By standard properties of integrals we have

$$
\left\|\boldsymbol{R}_{h}(\boldsymbol{\gamma})-\boldsymbol{R}_{h}(\boldsymbol{\beta})\right\| \leq \max _{0 \leq u \leq s_{4}}\left\|\left(\boldsymbol{f} \circ \phi_{\boldsymbol{\gamma}}\right)^{(5)}(u)-\left(\boldsymbol{f} \circ \phi_{\boldsymbol{\beta}}\right)^{(5)}(u)\right\| .
$$

From Lemma 10 we know that $\left(\boldsymbol{f} \circ \phi_{\boldsymbol{\alpha}}\right)^{(5)}(u)$ depends smoothly on $\boldsymbol{\alpha}$. The mean value theorem then gives

$$
\left\|\left(\boldsymbol{f} \circ \phi_{\boldsymbol{\gamma}}\right)^{(5)}(u)-\left(\boldsymbol{f} \circ \phi_{\boldsymbol{\beta}}\right)^{(5)}(u)\right\| \leq K\|\boldsymbol{\gamma}-\boldsymbol{\beta}\|
$$

for some constant $K$ that only depends on $w$ and $H$, and values of $\boldsymbol{f}^{(6)}$ in a neighborhood of $[0, h]$.

A similar argument can be applied to (42), resulting in a similar inequality. Taking determinants with $\boldsymbol{f}^{\prime}(0)$ and $\boldsymbol{f}^{\prime \prime}(0)$ and then assembling gives (44).

From Lemma 18 we see that the system of equations (41)-(42) can be written

$$
\boldsymbol{T}(\alpha)=\boldsymbol{d}-h \boldsymbol{s}_{h}(\boldsymbol{\alpha}) .
$$

If we know that the mapping $\boldsymbol{T}$ is invertible in a neighborhood of a solution, this suggests the fixed point iteration

$$
\boldsymbol{\alpha}_{k+1}=\boldsymbol{T}^{-1}\left(\boldsymbol{d}-h \boldsymbol{s}_{h}\left(\boldsymbol{\alpha}_{k}\right)\right)
$$

as a numerical method for finding the solution. To ensure that the iteration converges, we need $\boldsymbol{T}$ to be "nice", for example to have a nonsingular Jacobian at the solution. What we did in applying the implicit function theorem is essentially equivalent to this. However, the one case (38) where we have not proved Theorem 17 is characterized by the fact that the Jacobian is singular, and this is the reason why the implicit function theorem could not help us. A way out is provided by the following lemma. 
Lemma 19. Suppose that $d_{1,2} \neq 0$ and $\boldsymbol{f}^{\prime \prime \prime}(0)=0$, and also that $d_{1,4}=0$ and $5 d_{2,4}+d_{1,5}=0$. Consider the mapping $\boldsymbol{T}_{h}(\boldsymbol{\alpha})=\boldsymbol{T}(\boldsymbol{\alpha})+h \boldsymbol{E}(\boldsymbol{\alpha})$, where

$$
\boldsymbol{E}(\boldsymbol{\alpha})= \begin{cases}\left(d_{1,2} \alpha_{4}, 0,0,0\right) & \text { if } d_{1,5} \neq 0, \\ \left(0,0,0, d_{1,2} \alpha_{5}\right) & \text { otherwise. }\end{cases}
$$

This mapping has an inverse for $h<1$. If $\boldsymbol{f}^{(5)}(0) \neq 0$, this inverse satisfies, for $h>0$, the inequality

$$
\left\|\boldsymbol{T}_{h}^{-1}(\boldsymbol{b})-\boldsymbol{T}_{h}^{-1}(\boldsymbol{c})\right\| \leq C_{2} h^{-2 / 3}\|\boldsymbol{b}-\boldsymbol{c}\|,
$$

for all $\boldsymbol{b}$ and $\boldsymbol{c}$ in a sufficiently small neighborhood of $\boldsymbol{d}$. Here, the symbol $C_{2}$ denotes some constant independent of $h$.

Proof. Suppose that $d_{1,5} \neq 0$. Then the system $\boldsymbol{T}_{h}(\gamma)=\boldsymbol{c}$ is

$$
\begin{aligned}
\gamma_{4} d_{1,2}+\gamma_{4} h d_{1,2} & =c_{1}, \\
\left(4 \gamma_{3}+3 \gamma_{2}^{2}\right) d_{1,2} & =c_{2}, \\
\gamma_{5} d_{1,2}-10 \gamma_{2} d_{2,4} & =c_{3}, \\
\left(10 \gamma_{2} \gamma_{3}+5 \gamma_{4}\right) d_{1,2} & =c_{4} .
\end{aligned}
$$

We can determine $\gamma_{4}$ from the first equation and solve for $\gamma_{3}$ in the second equation, since $d_{1,2} \neq 0$. Inserting these values in the last equation, we end up with the cubic equation

$$
p_{h}\left(\gamma_{2} ; \boldsymbol{c}\right)=-15 d_{1,2} \gamma_{2}^{3}+5 \gamma_{2} c_{2}+2\left(5 c_{1}-c_{4}-h c_{4}\right) /(1+h)=0
$$

for $\gamma_{2}$. From this we can determine $\gamma_{2}$, provided $h \neq-1$ and $d_{1,2} \neq 0$. When $\gamma_{2}$ is known, we can determine $\gamma_{5}$ from the third equation, once again since $d_{1,2} \neq 0$.

We now study the system in the special case where the right-hand side is given by $\boldsymbol{d}=\left(d_{2,4},-d_{1,4}, d_{2,5},-d_{1,5}\right)$. If we denote the vector of unknowns in this case by $\boldsymbol{\alpha}$, the cubic equation reduces by (38) to

$$
p_{h}\left(\alpha_{2} ; \boldsymbol{d}\right)=-15 d_{1,2} \alpha_{2}^{3}+2 h d_{1,5} /(1+h)=0,
$$

so the solution is

$$
\alpha_{2, h}=\left(\frac{2 d_{1,5}}{15 d_{1,2}(1+h)}\right)^{1 / 3} h^{1 / 3}=K_{h} h^{1 / 3},
$$

where $K_{h}$ remains bounded for $h \in[0,1]$. From this we see that the derivative of $p_{h}$ with respect to $\alpha_{2}$, at the solution $\alpha_{2, h}$, is

$$
p_{h}^{\prime}\left(\alpha_{2, h} ; \boldsymbol{d}\right)=-45 d_{1,2} K_{h}^{2} h^{2 / 3} .
$$

Since this is nonzero, we know from the implicit function theorem that if we vary the right-hand side of (47) in a small neighborhood of $\boldsymbol{d}$, then there is always a solution of the corresponding cubic equation that depends smoothly on the righthand side. Therefore, for any right-hand side $\boldsymbol{b}$ in some small ball $B(\boldsymbol{d} ; w)$ around $\boldsymbol{d}$ with radius $w$, any $\eta$ sufficiently close to $\alpha_{2, h}$, and any $h \leq 1$, we have

$$
\left|p_{h}^{\prime}(\eta ; \boldsymbol{b})\right| \geq K_{1} h^{2 / 3},
$$

for some positive constant $K_{1}$ independent of $h$. 
Now let $\boldsymbol{b}$ and $\boldsymbol{c}$ be two vectors in the neighborhood $B(\boldsymbol{d} ; w)$, and let $\boldsymbol{\beta}$ and $\boldsymbol{\gamma}$ be vectors such that $\boldsymbol{T}_{h}(\boldsymbol{\beta})=\boldsymbol{b}$ and $\boldsymbol{T}_{h}(\boldsymbol{\gamma})=\boldsymbol{c}$. From the above we then have $p_{h}\left(\beta_{2} ; \boldsymbol{b}\right)=0$ and $p_{h}\left(\gamma_{2} ; \boldsymbol{c}\right)=0$, and therefore by the mean value theorem

$$
p_{h}^{\prime}(\eta ; \boldsymbol{b})\left(\gamma_{2}-\beta_{2}\right)=p_{h}\left(\gamma_{2} ; \boldsymbol{b}\right)-p_{h}\left(\beta_{2} ; \boldsymbol{b}\right)=p_{h}\left(\gamma_{2} ; \boldsymbol{b}\right)-p_{h}\left(\gamma_{2} ; \boldsymbol{c}\right),
$$

for some $\eta$ in $\left(\beta_{2}, \gamma_{2}\right)$ (or $\left.\left(\gamma_{2}, \beta_{2}\right)\right)$. Since $p_{h}(\boldsymbol{\gamma} ; \boldsymbol{c})$ is a polynomial in the right-hand side $\boldsymbol{c}$, we have

$$
\left\|p_{h}\left(\gamma_{2} ; \boldsymbol{b}\right)-p_{h}\left(\gamma_{2} ; \boldsymbol{c}\right)\right\| \leq K_{2}\|\boldsymbol{b}-\boldsymbol{c}\|
$$

for all $\boldsymbol{b}$ and $\boldsymbol{c}$ in $B(\boldsymbol{d} ; w)$, with $K_{2}$ some constant that only depends on $\boldsymbol{d}$ and $w$. Combining this with (49), we find that

$$
\left|\gamma_{2}-\beta_{2}\right| h^{2 / 3} \leq\left(K_{2} / K_{1}\right) \| \boldsymbol{c}-\boldsymbol{b}|| .
$$

Since the other variables depend linearly on $\beta_{2}$ and $\gamma_{2}$, we therefore end up with (46).

The above argument is clearly dependent on $d_{1,5}$ being nonzero. If $d_{1,5}=0$, then $d_{2,4}=0$ also, but if $\boldsymbol{f}^{(5)}(0) \neq 0$, then $d_{2,5} \neq 0$. Proceeding as above with the other definition of $E$, we end up with the same type of estimates, with $d_{2,5}$ taking over the role of $d_{1,5}$ in (48). If $d_{2,5}=0$ also, we would have $d_{1,4}=d_{2,4}$ as well as $d_{1,5}=d_{2,5}=0$, and hence $\boldsymbol{f}^{(3)}=\boldsymbol{f}^{(4)}=\boldsymbol{f}^{(5)}=0$, see below.

We can now solve the system (43) via fixed point iteration.

Lemma 20. Suppose that $d_{1,4}=0$ and $5 d_{2,4}+d_{1,5}=0$. If $h$ is sufficiently small and $\boldsymbol{\alpha}_{0}$ is sufficiently close to the solution $\boldsymbol{\alpha}^{*}$ of the Taylor scheme at $t=0$, then the fixed point iteration

$$
\boldsymbol{\alpha}_{k+1}=\boldsymbol{L}_{h}\left(\boldsymbol{\alpha}_{k}\right)=\boldsymbol{T}_{h}^{-1}\left(\boldsymbol{d}-h \boldsymbol{s}_{h}\left(\boldsymbol{\alpha}_{k}\right)+h \boldsymbol{E}\left(\boldsymbol{\alpha}_{k}\right)\right)
$$

converges to a solution of the system (43).

Proof. The lemma follows from the Banach fixed point theorem if we can show that $\boldsymbol{L}_{h}$ is a contraction. For this we note that for small $h$,

$$
\begin{aligned}
\left\|\boldsymbol{L}_{h}(\boldsymbol{\gamma})-\boldsymbol{L}_{h}(\boldsymbol{\beta})\right\| & \leq C_{2} h^{-2 / 3} \cdot h\left\|\boldsymbol{s}_{h}(\boldsymbol{\gamma})-\boldsymbol{s}_{h}(\beta)+\boldsymbol{E}(\boldsymbol{\beta})-\boldsymbol{E}(\boldsymbol{\gamma})\right\| \\
& \leq \tilde{C} h^{1 / 3}\|\boldsymbol{\gamma}-\boldsymbol{\beta}\|,
\end{aligned}
$$

since the mappings $\boldsymbol{s}_{h}, \boldsymbol{E}$ and $\boldsymbol{T}_{h}^{-1}$ satisfy Lipschitz conditions, see Lemma 18 and Lemma 19. Also, if we let $\boldsymbol{\alpha}^{*}$ denote the solution in the Taylor case, so that $\boldsymbol{T}\left(\boldsymbol{\alpha}^{*}\right)=\boldsymbol{d}$ and $\boldsymbol{T}_{h}\left(\boldsymbol{\alpha}^{*}\right)=\boldsymbol{d}+h \boldsymbol{E}\left(\boldsymbol{\alpha}^{*}\right)$, we find

$$
\begin{aligned}
\left\|\boldsymbol{L}_{h}(\boldsymbol{\gamma})-\boldsymbol{\alpha}^{*}\right\| & =\left\|\boldsymbol{L}_{h}(\boldsymbol{\gamma})-\boldsymbol{T}_{h}^{-1}\left(\boldsymbol{T}\left(\boldsymbol{\alpha}^{*}\right)+h \boldsymbol{E}\left(\boldsymbol{\alpha}^{*}\right)\right)\right\| \\
& \leq h^{1 / 3}\left(K_{1}\left\|\boldsymbol{\gamma}-\boldsymbol{\alpha}^{*}\right\|+\left\|\boldsymbol{s}_{h}(\boldsymbol{\gamma})\right\|\right) \\
& \leq h^{1 / 3}\left(K_{1}\left\|\boldsymbol{\gamma}-\boldsymbol{\alpha}^{*}\right\|+K_{2}\right),
\end{aligned}
$$

for small $h$, where the last inequality follows since $\boldsymbol{s}_{h}(\gamma)$ depends smoothly on $\boldsymbol{\gamma}$. Therefore we have

$$
\left\|\boldsymbol{L}_{h}(\boldsymbol{\gamma})-\boldsymbol{\alpha}^{*}\right\| \leq\left\|\boldsymbol{\gamma}-\boldsymbol{\alpha}^{*}\right\|
$$

for sufficiently small $h$, so that $\boldsymbol{L}_{h}$ maps any sufficiently small neighborhood of $\boldsymbol{\alpha}^{*}$ into itself. We therefore conclude that $\boldsymbol{L}_{h}$ is a contraction for small $h$ and therefore has a fixed point, which is the solution of (43). 
Proof of Theorem 17. The case where $\boldsymbol{f}^{(i)}(0)=0$ for $i=3,4,5$ is not covered by the above. But in this case we have

$$
\boldsymbol{f}(t)=\boldsymbol{f}(0)+\boldsymbol{f}^{\prime}(0) t+\boldsymbol{f}^{\prime \prime}(0) t^{2} / 2+\boldsymbol{f}^{(6)}(0) t^{6} / 6+\cdots
$$

so that the initial quadratic part of $\boldsymbol{f}$ will be a sixth-order accurate approximation in a neighborhood of $t=0$.

\section{Conclusion}

In this paper we have introduced a general framework for parametric interpolation by polynomial curves. This generalizes a number of parametric interpolation methods that have appeared in recent years and suggests a host of new schemes. We have proved existence of solution and high-order convergence for the simplest schemes in each space dimension, and also for cubic schemes in the plane.

From our results here it is easy to make general conjectures. In the plane, the conjecture is that there is always a polynomial curve of degree $n$ interpolating $\boldsymbol{f}$ with approximation order $2 n$ in a neighborhood of a point where the first $n$ derivatives span $\mathbb{R}^{2}$. A completely analogous conjecture can be formulated in general space dimension.

To prove such a conjecture seems like a very difficult task. One natural approach is to start with the Taylor case and then use perturbation arguments like we did here. However, even in the Taylor case the equations very quickly become very messy. There would be some hope if the polynomial equations could be reduced to one equation in one unknown of odd degree, as in the cubic case when $d_{1,2} \neq 0$, since we then always have a real solution. But experiments in Mathematica with quartic approximations in the plane show that the equations can only be reduced to an equation of even degree. The only hope of success seems to be to attack the problem with other methods. Indeed, the equations can be formulated easily in terms of divided differences and the conditions for solvability seem simple; there

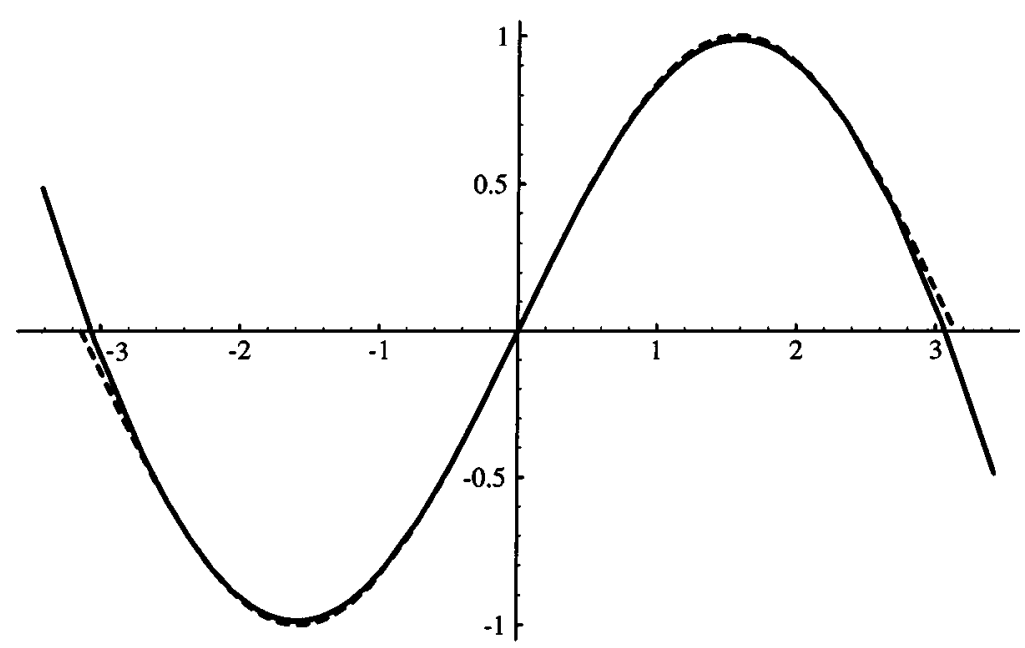

Figure 1. Cubic Hermite interpolation to the parametric curve $(t, \sin t)($ dashed) at the points $(-3 \pi / 4,-3 \pi / 4,0,0,3 \pi / 4,3 \pi / 4)$ 
is therefore hope that one can get to a solution without having to consider each equation in detail. For examples of parametric interpolation methods we refer the reader to the references, where many nice illustrations can be found. However, numerical experiments confirm our results, and we include one example which illustrates the potential of the schemes. In Figure 1 we have approximated the curve $(t, \sin t)$ by the cubic Hermite interpolant at three points near the origin. Numerically, we can let all the points approach $t=0$, and in this process the error is reduced by a factor of about 64 each time the length of the interpolation interval is halved, as is predicted by Theorem 17. Note that the curvature of this curve satisfies $\kappa(0)=0$, so that $\left\langle\boldsymbol{f}^{\prime}(0), \boldsymbol{f}^{\prime \prime}(0)\right\rangle=0$. It is therefore a curve that is not covered by the convergence analysis in [2].

\section{REFERENCES}

1. R. Bartels, J. Beatty, B. Barsky (1987): An Introduction to Splines for Use in Computer Graphics and Geometric Modeling. Los Altos, California: Morgan Kaufmann Publishers Inc. MR 89f: 65018

2. C. de Boor, K. Höllig, M. Sabin (1988): High accuracy geometric Hermite interpolation, Comput. Aided Geom. Design, 4:269-278. MR 90b:65014

3. W. L. F. Degen (1992): Best approximation of parametric curves by splines. In: Mathematical Methods in Computer Aided Geometric Design II (T. Lyche, L. L. Schumaker, eds.). San Diego: Academic Press, pp. 171-184. MR 93e:41017

4. L. F. Degen (1993): High accurate rational approximation of parametric curves. Comput. Aided Geom. Design, 10:293-313. MR 94i:65022

5. T. Dokken, M. Dæhlen, T. Lyche, K. Mørken (1990): Good approximation of circles by curvature continuous Bézier curves. Comput. Aided Geom. Design, 7:33-41. MR 91j:65028

6. E. F. Eisele (1994): Chebychev approximation of plane curves by splines, J. Approx. Theory, 76: $133-148$. MR 95c: 41024

7. G. Farin (1993): Curves and Surfaces for CAGD. A Practical Guide. Third edition. Cambridge, Massachusetts: Academic Press Inc. MR 93k:65016

8. M. Floater (1994): An $O\left(h^{2 n}\right)$ Hermite approximation for conic sections. Preprint, SINTEFSI, Oslo.

9. T. N. T. Goodman (1985): Properties of $\beta$-splines. J. Approx. Theory, 44:132-153. MR 87f: 41020

10. J. A. Gregory (1989): Geometric continuity. In: Mathematical Methods in CAGD (T. Lyche, L. L. Schumaker, eds.). Boston: Academic Press, pp. 353-371. MR 91d:65033

11. R. Klass (1983): An offset spline approximation for plane cubic splines. Comp. Aided Design, 15:297-299.

12. D. E. Knuth (1975): The Art of Computer Programming. Vol. 1, Fundamental Algorithms. Reading, Massachusetts: Addison Wesley Publishing Company. MR 51:14624

13. M. A. Lachance, A. J. Schwartz (1991): Four point parabolic interpolation. Comput. Aided Geom. Design, 8:143-149. MR 92a:65045

14. T. Lyche, K. Mørken (1994): A metric for parametric approximation. In: Curves and Surfaces in Geometric Design (P. J. Laurent, A. LeMéhauté, L. L. Schumaker, eds.). Wellesley, Massachusetts: A K Peters, Ltd., pp. 311-318. MR 95g:65027

15. K. Mørken (1991): Best approximation of circle segments by quadratic Bézier curves. In: Curves and Surfaces (P. J. Laurent, A. Le Méhauté, L. L. Schumaker, eds.). Boston: Academic Press, pp. 331-336. CMP 91:17

16. K. Mørken (1995): Parametric interpolation by quadratic polynomials in the plane. In: Mathematical Methods for Curves and Surfaces. Vanderbilt Univ. Press, Nashville, TN, pp. 385-402. MR 96h:65023

17. A. Pinkus (1993): Uniqueness in vector valued approximation. J. Approx. Theory, 73:17-93. MR 94e: 41047

18. A. Rababah (1992): Approximation von Kurven mit Polynomen und Splines. Ph. D. thesis, Mathematisches Institut A, Universität Stuttgart. 
19. A. Rababah (1995): High order approximation method for curves. Comput. Aided Geom. Design, 12:89-102. MR 95m:65030

20. R. Schaback (1989): Interpolation in $\mathbb{R}^{2}$ by piecewise quadratic visually $C^{2}$ Bézier polynomials. Comput. Aided Geom. Design, 6:219-233. MR 90j:65020

21. R. Schaback (1989): Convergence of planar curve interpolation schemes. In: Approximation Theory VI (C. Chui, L. L. Schumaker, J. Ward, eds.). Boston: Academic Press, pp. 581-584. CMP 91:07

22. R. Schaback (1993): Planar curve interpolation by piecewise conics of arbitrary type. Constr. Approx., 9: 373-389. MR 94h:41006

23. B. Sendov (1969/70): Parametric Approximation. Annuaire Univ. Sofia Fac. Math., 64:237247. MR 46:7771

24. M. Spivak (1979): A Comprehensive Introduction to Differential Geometry, Volume II. Second Edition. Wilmington, Delaware: Publish or Perish Inc. MR 82g:53003b

25. J. Szabados (1972): On parametric approximation. Acta Math. Sci. Hung., 23:275-287. MR 52:8732

Department of Informatics, University of Oslo, P. O. Box 1080 Blindern, N-0316 OSLO, NORWAY

E-mail address: knutm@ifi.uio.no

Institut für Angewandte Mathematik, Universität Bonn, Wegelerstr. 6, D-53115 Bonn, Germany

E-mail address: unm11c@ibm.rhrz.uni-Bonn.de 